\title{
Webster et Robert Merle: Du Démon blanc à Flamineo : revisiter le théâtre de l'effroi
}

\section{Anne Wattel}

\section{Q OpenEdition \\ 1 Journals}

\section{Édition électronique}

URL : https://journals.openedition.org/shakespeare/4097

DOI : 10.4000/shakespeare.4097

ISSN : 2271-6424

Éditeur

Société Française Shakespeare

\section{Référence électronique}

Anne Wattel, « Webster et Robert Merle: Du Démon blanc à Flamineo : revisiter le théâtre de l'effroi », Actes des congrès de la Société française Shakespeare [En ligne], 36 | 2018, mis en ligne le 19 février 2018, consulté le 25 août 2021. URL : http://journals.openedition.org/shakespeare/4097 ; DOI : https://doi.org/10.4000/shakespeare.4097

Ce document a été généré automatiquement le 25 août 2021.

(c) SFS 


\title{
Webster et Robert Merle: Du Démon blanc à Flamineo : revisiter le théâtre de l'effroi
}

\author{
Anne Wattel
}

1 Robert Merle, après son premier roman, Week-end à Zuydcoote, récit de la débâcle de 1940 qui lui valut le prix Goncourt, entreprend en 1950 de retracer «la lente et tâtonnante mise au point de l'usine de mort d'Auschwitz ${ }^{1}$ ». Il explore, sidéré, au Centre de Documentation Juive International toutes les archives de Nuremberg et il essaie d'appréhender Rudolf Hœss, le bourreau d'Auschwitz, cet homme, un homme, qui tua deux millions et demi de ses semblables et auquel il choisit dans son roman, La mort est mon métier, paru en 1952, de donner une voix, à la première personne. Et pour comprendre, pour saisir le pire du $\mathrm{xx}^{\mathrm{e}}$ siècle, pour saisir le mal et aller au-delà de la sidération, de l'effroi, Merle, professeur de littérature anglaise, fait d'abord un détour par le XVII siècle, par le théâtre de l'effroi, non pas par Shakespeare qu'il connaît pourtant très bien, mais par Webster et sa pièce de 1612, Le Démon blanc ${ }^{2}$. Il se tourne vers le dramaturge dont il estime qu'il est un « maître ès agonies » qui a exploré la mort de manière obsessionnelle, avec "une force étrange", "avec des accents plus inoubliables que ceux de Shakespeare» (DB, 72). Il se tourne vers le morbide et le macabre, vers l'univers de Webster chez lequel «le "mal" atteint sa plus grande profondeur » (DB, 68).

2 L'œuvre, on le sait, s'inspire d'un épisode réel, l'histoire de Vittoria Accoramboni, qui défraya la chronique italienne entre 1580 et $1585^{3}$. Il s'agit d'une tragédie de vengeance qui conte les amours adultères du duc Brachiano et de la belle Vittoria qui, épaulés par un entremetteur, le frère de cette dernière, Flamineo, s'emploient à éliminer les obstacles à leur bonheur et font assassiner et l'époux fantoche de Vittoria, et la vertueuse Isabella, sœur de Francisco de Médicis, duc de Florence, qui obtiendra vengeance.

3 On peut véritablement parler de fascination de Merle pour cette pièce de Webster et cette chronique italienne: non seulement il la traduit en 1950 aux éditions Aubier 
Bilingue, mais il la réécrit aussi avec son Flamineo, mis en scène pour la première fois au théâtre de Rennes en 1949 ; elle lui inspire également trois versions romancées publiées entre 1958 et $1987^{4}$.

4 Nous nous attarderons tout d'abord sur la pièce de Webster et sur le fait qu'au-delà des délices macabres de la pièce baroque, ce que Merle admire, c'est la peinture d'un univers en totale décomposition, peuplé d'êtres effroyables, mais non pas de monstres. Car ces êtres-là, les Brachiano, les Vittoria, les Lodovico et Flamineo sont, certes, des criminels qui font frémir, mais ce sont des hommes dont la part démoniaque trouve à s'exprimer dans une société qui a quelque chose de pourri en elle. Et c'est cette peinture inégalée, moderne, intemporelle du mal que Merle trouve en germe chez Webster.

5 C'est donc à partir de Webster, nourri de Webster, par-delà Webster, que Merle écrit son Flamineo ${ }^{5}$, un Flamineo qui va le guider dans son exploration du bourreau du $\mathrm{xx}^{\mathrm{e}}$ siècle, de Rudolf Hœss, un Flamineo qui devient " une pièce d'aujourd'hui ${ }^{6}$ ", comme le notait Aragon, une pièce centrée sur un personnage, le tueur, et sur cette question qui traverse en filigrane toutes les œuvres de Merle : d'où vient que nous fassions le mal ?

\section{Le Démon blanc, une pièce de l'effroi}

Webster est considéré comme un maître de l'épouvante : il aurait engendré, selon Henri Fitzjeffrey, son contemporain qui en fit un portrait à charge en 1617 dans ses Notes $d u$ Théâtre des Blackfryers, une œuvre monstrueuse, «some centaur strange, some huge Bucephalus / or Pallas, sure, [.... $]^{7}$ ». Pour Philarète Chasles, professeur au collège de France, du XIX siècle, "la Gorgone est sa muse; il se plaît dans l'horrible»; et l'universitaire de préciser : "Ne cherchez rien de tendre, de gracieux ou de tempéré dans ces tragédies, désert sanglant où règne une atmosphère empoisonnée, où le forfait appelle le forfait $[. . .]^{8}$ ». Plus récemment, Yves Peyré, dans sa notice pour l'édition de la Pléiade, évoquant l'univers du Démon blanc, indique qu'il est «monstrueux et contre nature ${ }^{9} "$.

Détracteurs ou admirateurs, tous s'accordent à dire que Webster est l'auteur d'une œuvre-centaure, qui peint l'inhumanité tapie au fond de l'homme, la sauvagerie ; d'une œuvre qui relève de l'excès ; une œuvre-gorgone qui méduse, pétrifie le spectateur.

Et, il est vrai, il s'agit bien là d'un théâtre de l'effroi, un théâtre plein « d'horreur et de sang» (DB, I. i.81), fait pour les yeux et sur lequel s'amoncellent les corps meurtris, corps douloureux qui agonisent longuement. Il faut ensanglanter la scène; pas moins de sept morts sont offertes en spectacle.

On note chez Webster un indéniable raffinement dans la monstration de l'horreur, une monstration renforcée par la mise en scène d'un voyeurisme de l'acte criminel. Ce voyeurisme peut être macabre et jouissif, comme c'est le cas pour le duc Brachiano qui réclame qu'on les lui montre, ces meurtres, qu'on lui offre la vision de leur exécution (DB, II.ii.131). La magie est alors convoquée et apparaissent sous ses yeux deux pantomimes au fort potentiel dramatique. Brachiano, assistant en qualité de spectateur à la mort de sa propre épouse qu'il a commanditée, s'exclame : « Fort bien, donc elle est morte » (DB, II.ii.133) : simple constat, cynique et glaçant.

10 Avec le personnage de Cornelia, la mère pathétique, le voyeurisme se fait frémissant. Webster ne se contente pas de mettre en scène le fratricide, il pose l'acte sous les yeux 
de la mère impuissante et le spectateur, ébranlé par l'acte lui-même, l'est d'autant plus qu'il voit la mère horrifiée par Flamineo, le monstre qu'elle a enfanté ; qu'il découvre l'horreur d'une mère qui voit dans son fils l'entremetteur et qui s'exclame : «plût à Dieu que je ne t'eusse pas enfanté ! » (DB, I.ii.103).

L'effroi dans la pièce de Webster, vient aussi des mots eux-mêmes : la parole sadique, la violence du langage se font l'écho d'un désir de destruction et profanation du corps de l'ennemi car la mort ne suffit pas. Lodovico, artiste en cruauté, qui rêve de faire avec les tripes de ses ennemis « de la dentelle italienne » (DB, I.i.81), contemple à la fin de la pièce la scène qu'il vient d'ensanglanter triplement et se félicite: «Je me glorifie de dire : c'est moi qui ai fait ceci [...] - c'est moi qui ai peint cet effet de nuit. C'est mon chef d'œuvre " (DB, V. 6. 275). Tous les personnages ou presque s'adonnent à des fantasmes barbares : on rêve de "hacher menu » et laisser " le sauvage vent du nord balayer les morceaux " (DB, IV.ii.183); d'empaler sur un piquet ou un pal (DB, V.i.219; V.vi.265); on souhaite que l'autre s'étouffe avec sa salive (DB, III.ii.159); on se déclare prêt à s'arracher la lèvre d'un coup de dent plutôt que de donner un baiser (DB, IV.ii. 189). Et même la pure Isabella, épouse de Brachiano, évoque, dans une cascade d'infinitifs que ne renierait pas Médée, ses désirs de vengeance : «Arracher les yeux de la catin, la laisser mourir à petit feu quelque vingt mois, lui couper le nez et les lèvres, arracher ses dents pourries, et faire momifier sa chair pour la garder comme un trophée de ma juste colère " (DB, II.i.121). Mutilations, dépouilles profanées, corps percés, hachés, empalés, par le feu, par le fer, par le pieu : chacun semble rivaliser d'inventivité quand il s'agit d'évoquer la torture.

Mort et putréfaction envahissent également l'arrière-plan. Tout dans cette pièce est corrompu : corrompues, la théologie (DB, IV.i.179), l'époque (III.ii.157), la femme; violée, la justice. Le Démon blanc est une œuvre de la pourriture, et rien n'échappe à la décomposition : le corps se dégrade, dents et membres sont pourris (DB, IV.ii.189, II.i. 121); la nature se meurt avec son gui flétri et épuisé (DB, II.i.129); le médecin est un " habile et corrompu bourreau " (DB, V.iii.229). Vie et mort coexistent tant et si bien qu'elles n'en sont plus antagonistes: la vie, faisandée, n'est qu'une étape vers la mort. Cette pièce est bien, comme l'indique Yves Peyré une "tragédie de la dislocation du sens, de l'éclatement des perspectives et de la perversion des valeurs ${ }^{10}$ ».

Le baroquisme de Webster est fréquemment présenté de manière critique: il proposerait une surenchère dans l'effroi et la monstration, il se complairait dans le sang qui appelle le sang, d'où l'accusation bien postérieure d'être, comme le notait Marc Beigbeder dans Les Lettres Françaises en 1953, un «bâtisseur de mélo » mettant en scène un « Hamlet des bas-fonds ${ }^{11}$ ".

14 Merle, qui analyse longuement la pièce dans son introduction aux éditions Aubier, s'arrête sur les reproches qui ont pu être faits à Webster, à commencer par celui d'avoir négligé, malmené l'Histoire. En appliquant les lois de "la concentration et de l'accumulation dramatique ", en transformant l'Histoire en histoire de vendetta, en accumulant les menaces qui pèsent sur les deux amants, Webster a, selon Merle, " pimenté » l'histoire et l'a rendue "scéniquement plus efficace» (DB, 20). Si l'on compare, dit-il, «la version historique des faits et la version de Webster, on ne peut manquer d'apercevoir combien, pour les effets de "la pitié et de la terreur" celle-ci l'emporte sur celle-là » (DB, 20-21).

15 Merle récuse le terme de "mélodrame ». Selon lui la différence entre drame et mélo réside « dans le fait que le mélo s'organise et se construit autour du frisson d'horreur - 
du "thrill" - tandis que le "thrill" n'est qu'un des éléments auxquels le drame peut ou non recourir " (DB, 27). «Le mélodrame - le "thrill" pour l'amour seul du "thrill" - est une erreur esthétique. Mais le morbide est du domaine légitime de l'art» (DB, 28). Par ailleurs, selon Merle :

Empoisonnements raffinés, guets-apens mortels, meurtres maquillés en accidents, massacres et savantes tortures, fantômes et mandragores, rien de tout cela, en fait, ne paraissait aux Élisabéthains incroyable ou excessif. Au XvI siècle, ce que nous appelons le « mélodrame » c'était l'étoffe même dont la vie était faite. (DB, 26)

Et cette étoffe, cet arrière-plan des tragédies du sang, force est de constater, comme le notait Aragon - qui s'étonnait de l'accueil fait à Webster dans Les Lettres Françaises -, qu'il est curieux "qu'elles semblent être devenues incompréhensibles aux contemporains du nazisme, à ceux qui ont vu fumer les fours d'Auschwitz [... $]^{12} »$.

\section{Une peinture inégalée du mal}

17 Si Merle reprend dans son Flamineo cet arrière-plan, s'il s'adonne au morbide et parsème sa scène de cadavres, ce n'est pourtant pas là ce qui explique son détour par Webster. Par dessus tout, c'est la peinture inégalée du mal que Webster propose qui le fascine.

L'œuvre, dès son titre, est placée sous le signe du démon; pourtant, étonnamment, elle ne met en scène que des êtres humains, terriblement humains. Pas de sorcières, ni de véritable diable en scène : les termes " enfer, démon, sorcière " s'ils apparaissent bien dans la pièce, ne sont que métaphores. Le mal est bien présent, mais c'est un mal humanisé, au cœur de la nature humaine.

Dans la pièce de Webster, on fait à peine trembler le spectateur avec l'au-delà. Le Diable n'est, comme le dit Vittoria que « carton peint » juste bon à faire peur aux enfants (DB, III.ii.151), or elle a passé l'âge des tremblements sans cause. Au mieux, il relève du délire, celui d'un Brachiano agonisant, empoisonné (DB, V.iii.233) qui voit le diable. Délire que tout cela, comme l'indique Vittoria qui répond laconiquement: « Monseigneur, il n'y a rien ici».

20 Il en est de même pour les spectres ${ }^{13}$ : Webster, fidèle à la tradition, ne manque pas cette scène à faire : deux spectres sont convoqués, ceux d'Isabella (DB, IV.i.179) et de Brachiano (V.iv.253-255). Le premier est évacué par la raison : il n'émerge que par le pouvoir de l'imagination, comme le constate Francesco de Médicis, le frère d'Isabella: « il n'y a rien d'autre ici que ma mélancolie ». Spectres et diables, tout cela n'est que "conte de bonne femme» (DB, IV.i.179), hallucinations émanant d'esprits mélancoliques ou perturbés. Le second, le spectre de Brachiano qui apparaît à Flamineo suscite il est vrai plus d'effroi. C'est une "terrible apparition", "davantage qu'un simple accès d'hypocondrie » (DB, V.iv.255), mais c'est un "risible objet », drôlement attifé et qui n'effraie que parce que Flamineo y voit un présage funeste.

21 L'enfer est bien sûr évoqué : mais il devient fait de langage, manière d'imprécation et permet de montrer la supériorité de l'humain dans la noirceur, dans la douleur, dans l'horreur: «l'enfer n'est que rosée de glace, comparée à mon affliction » dit Isabella (DB, II.i.121). Et si l' " odeur de suie, le foie bouilli, la brûlure des flammes infernales » sont bien évoqués (DB, V.vi.265), c'est uniquement lorsque Flamineo feint de mourir, c'est du chiqué... 

qu'il transforme le monde en enfer : «Quel enfer que ce lieu! » dit Vittoria (DB, V.iii. 239). Si démon il y a, c'est au cœur de l'homme. C'est cette nature humaine que peint Webster, avec sa galerie de personnages à la noirceur démoniaque qui horrifient le spectateur : son démon blanc, pire que le noir, c'est Vittoria, qui, sous de beaux atours cache une âme noire ; elle est une véritable pomme de Sodome, un «beau fruit » en apparence mais qu'on verrait "se réduire en suie et en cendres » à peine touché (DB, III.ii.145). Elle est un diable « incarné en dehors séduisants » (DB, III.ii.155), et c'est elle qui suggère à son amant « de faire disparaître son mari et la duchesse » (DB, I.ii.97), elle qui, corrompue, passe de l'adultère au crime, comme le dit le cardinal Monticelso lors de son procès : « À la suite du démon Adultère, le démon Meurtre apparaît » (DB, III.ii. 149). qui, ayant pris gout au sang, ne cessent de vouloir tuer" (DB, IV. 3. 208); c'est Brachiano, que Lodovico et ses comparses déguisés en moines lors de la scène d'assassinat, associent par trois fois au démoniaque (DB, V.iii.237-239) ; c'est Zanche, la mauresque, noire de peau, dépravée, corrompue ; et c'est bien sûr Flamineo, dont seul le diable sait quelles vilénies il pourrait commettre (DB, V.i.215); Flamineo le proxénète, le fratricide, qui injurie sa propre mère, et ne recule devant rien.

Nous l'avons dit, ces personnages voués au mal sont humains. Tous ont subi un échec d'intégration; ce sont des héros en lutte contre les obstacles à leurs désirs, et qui ne sauraient se soumettre. En cela, ils suscitent une admiration horrifiée. Ainsi, Brachiano et Vittoria sont deux mal-mariés et, si le duc fomente des actes criminels, il n'est, comme le dit Robert Merle «fondamentalement ni bas, ni cruel»; il «n'est pas un monstre, mais il est muré dans la prison de sa passion. Il est mort à tout ce qui n'est pas l'urgence de son amour » (DB, 36). Vittoria choisit la transgression et le rejet de l'ordre moral par amour, pour n'avoir pas à mettre de freins à ses désirs. «Elle fait face de façon superbe ", nous dit Merle (DB, 59). On le voit notamment lorsqu'elle récuse l'avocat qui s'exprime en latin puis dans un jargon inintelligible : «Je ne veux pas que votre accusation s'enveloppe de la brume d'une langue étrangère. Que tout le monde ici entende ce dont vous m'accusez » (DB, III.ii.143). Le démon blanc n'est pas hypocrite. Flamineo, l'abject, est le type même du malcontent, machiavélique ; c'est un héros de la frustration, un «être fier, qui [...] souffre d'un insatiable sentiment d'infériorité ou d'échec » $(\mathrm{DB}, 64)$ parce que sa pauvreté en fait un laquais, un chien. C'est un « Hamlet noir ", comme le désigne Merle, qui a le franc parler du railleur, même avec son maitre, et qui adopte une franchise brutale, refuse l'hypocrisie sociale. Il est celui qui pointe le désordre derrière l'ordre apparent, qui revendique sa responsabilité, qui défie. Les personnages de Webster, on le voit, sont les victimes de la situation et leur lutte pour que triomphent leurs objectifs ne manque pas de grandeur.

Chez Webster, Merle trouve donc une galerie de personnages, effroyablement humains, qui adoptent un comportement criminel dans des conditions particulières de dégénérescence de la société et parce qu'ils sont murés dans une idée fixe, l'amour, l'ambition. Ce qui interpelle Merle dans cette pièce c'est donc aussi une interrogation sur la fin et les moyens, cette possible porosité entre innocence et culpabilité, bourreaux et victimes; des questions qui ne sont pas, en 1950, sans faire écho au débat Sartre/Camus, aux justes et aux mains sales; ce qui interpelle Merle dans cette pièce, 
c'est l'horreur du mal - est-il radical? est-il banal? - alors même qu'il tente d'appréhender Rudolf Hœss.

\section{Du Démon blanc au Hamlet noir}

Lorsque Merle décide de réécrire Le Démon blanc, c'est sur le héros noir (DB, 69) ${ }^{14}$, sur "l'immonde, le sinistre, le fascinant Flamineo ", qu'il focalise son attention. L'auteur $\mathrm{du} \mathrm{xx}^{\mathrm{e}}$ siècle notait déjà dans son analyse de l'œuvre de Webster, le "pouvoir de fascination » du personnage de Flamineo (DB, 71). Il modifie alors le titre : au « démon blanc $^{15}$ ", Merle substitue un "Flamineo». Il simplifie les péripéties de la pièce élisabéthaine ${ }^{16}$, resserre l'action autour de son personnage éponyme et transforme les données de la pièce source de manière à ce que «tout ce qui est crime appartie[nne] à Flamineo $»^{17}$. C'est le tueur qu'il choisit d'ausculter dans son Flamineo. De cette différence qu'il jugeait essentielle, Aragon disait : « malgré la fable empruntée, c'est elle qui fait que Flamineo n'est plus une pièce de Webster, mais de Robert Merle ${ }^{18}$ ».

Certains critiques reprochèrent à Merle de s'être contenté d'élaguer Le Démon blanc et de n'en proposer qu'une redite. Or Merle a modifié «la physionomie du héros primitif $^{19}$ ", et inséré des thèmes nouveaux, ceux de la damnation, du monstre, de l'inceste. Ce sont ces modifications qui vont nous intéresser maintenant: ce choix de centrer l'œuvre sur le Hamlet noir plutôt que sur le démon blanc; les explications psychologiques que Merle ajoute à Webster; les chemins du meurtre qu'il explore au travers du personnage de Flamineo.

Ce que Merle explore dans ses œuvres romanesques ou théâtrales, c'est ce qui pousse l'homme à rechercher la mort de l'homme. Une phrase, une même phrase, revient comme un leitmotiv : cette phrase, en 1949, c'est celle de Maillat indiquant que la mort des hommes «la Nature s'en charge. C'est ignoble de lui donner un coup de main ${ }^{20}$ »; c'est celle aussi du Magicien qui tentant d'empêcher la mise à mort d'Isabelle dit à Brachiano : "Crois-moi, la Nature [...] tue bien assez vite [les créatures de Dieu]. La Nature est la grande tueuse. Et l'homme qui tue son frère entre avec elle dans une ignoble complicité » (FLAM, II.ii.73). Plus tard, en 1950, en en retrouve une variante dans Sisyphe et la Mort puis en 1957 dans le Nouveau Sisyphe.

Faire alliance, être complice, donner un coup de main, toutes ces expressions disent le lien étroit entre l'homme et le meurtre. Dans ses œuvres, Merle explore «l'ingéniosité fameuse que les Mortels ont toujours montrée à [...] servir [la Mort ${ }^{21} »$; il questionne leur amour pour la mort, leur désir de la mort des autres, leur consentement à leur propre mort et, ce faisant, il s'interroge après-guerre, après-Auschwitz, sur le mal historique et ce pour faire face à l'effroi. On comprend mieux pourquoi c'est chez Webster qu'il va puiser, Webster qu'il tenait pour un amoureux de la mort, un maitre ès agonies, un peintre du mal.

Plus encore que chez Webster, Merle montre la porosité entre bourreaux et victimes : le mal est tapi au cœur de la nature humaine et, dans certaines conditions, mêmes les plus vertueux, les plus consciencieux peuvent s'y vautrer. Ainsi, si l'Isabella de Webster est une figure touchante et sublime, si son Marcello, frère de Flamineo, est un des rares contrepoints vertueux de la pièce, chez Merle, tous deux sont d'effroyables criminels en germes. 
31 Isabelle incarne le sublime. Sublime son amour pur pour Brachiano, sublime son mensonge, lorsqu'elle feint devant son frère, duc de Médicis, de rompre de son propre chef avec Brachiano pour que la guerre n'éclate pas entre les deux hommes. La scène est directement reprise de Webster. L'intention est noble, mais Merle insère, après le jeu d'Isabelle un dialogue entre Brachiano et Flamineo qui analysent ce à quoi ils viennent d'assister. Et seul Flamineo voit que «tout n'était pas que feinte dans sa feinte " (FLAM, II.ii.69), il voit la possible meurtrière derrière le sublime, dans la noblesse, il voit le gluant. «Tenez, voyez ce fil! du sublime ! et avec ça, c'est gluant, le sublime, ça colle aux doigts, ça paralyse ! J'en suis tout entortillé, Monseigneur. Comme une mouche!» (FLAM, I.i.67). Isabelle, la vertueuse, est aussi capable, par amour, de passer à l'acte : c'est cette révélation qui la perdra.

Le personnage de Marcello, antithèse de son frère Flamineo, est lui aussi, chez Webster, une figure de vertueux ${ }^{22}$. Merle nous propose une peinture plus nuancée : Caïn et Abel sont plus semblables qu'on ne le croit. Car Marcello aussi est un tueur, qui fait peu de cas de la vie humaine, mais il tue par ordre, « consciencieux sans conscience ${ }^{23}$ »-sans mauvaise conscience - étant capitaine au service de Médicis. Ce sont des ennemis de l'État, des Turcs qu'il tue. Et en cela, ce criminel-là est respecté, c'est un héros quand son frère, lui, est un monstre. C'est là toute l'ironie de la chose que Flamineo, cynique, débusque : il y a des combats honorables, des meurtres justes, héroïques, vertueux, dignes d'admiration et des assassinats monstrueux qui suscitent l'effroi.

Dans la pièce de Merle, il n'y a pas, à proprement parler, de justes, il n'est plus guère possible de distinguer le bon grain de l'ivraie, de séparer les bourreaux des victimes. Tous ont les mains sales mais certains les exposent et d'autres les cachent : $s$ 'il est des monstres, des Lodovico, et, plus abjects encore, des Flamineo, ils ne sont pas les seuls coupables. Et c'est cette question de la culpabilité que Merle ajoute à Webster et qui apparaît clairement dans la modification de la pantomime, empruntée au texte source, qui donne à voir la mort d'Isabelle. Comme chez Webster, un magicien, dans Flamineo, fait apparaître la scène aux yeux de Brachiano. Comme chez Webster, ce dernier va alors être réduit au statut de spectateur, mais en 1612, on s'en souvient, une fois la scène faite, Brachiano s'exclamait : « Fort bien, donc elle est morte » (DB, II.ii. 133).

C'est tout différent chez Merle. Son magicien ne se limite pas à faire apparaître, il tente de détourner Brachiano et de sa recherche de mort et de sa curiosité morbide. En véritable metteur en scène, il a le pouvoir encore, avant que ne se lève le rideau, de "déjouer le piège infernal que Flamineo a préparé pour elle » (FLAM, II.ii.74). «Il est temps encore » répète-t-il à deux reprises (FLAM, II.ii.72; 74). Mais le Duc veut voir, il veut voir l'exécution de son ordre, il veut voir la mort d'Isabelle, il veut en jouir, persuadé que « la mort d'Isabelle ne [le] troublera pas davantage que celle d'un chien dans [s]on chenil » (FLAM, II. ii. 75). Après avoir exigé que le magicien mette en branle son pouvoir de faire voir, Brachiano, assistant au "spectacle", en chair, en corps, voudra y mettre fin, voudra dé-jouer ce qui s'annonce :

BRACHIANO. Giordano

LE MAGICIEN. Monseigneur?

BRACHIANO. Peux-tu encore, par ton art, retarder, dans son vol, la minute fatale?

LE MAGICIEN. Non, Monseigneur.

BRACHIANO. Si tu ne peux arrêter l'écoulement du temps, peux-tu du moins, par le pouvoir magique de tes formules, amputer la cause de son effet mortel?

LE MAGICIEN. Non, Monseigneur.

BRACHIANO. Mais tu peux encore, n'est-ce pas, Giordano, tu peux arracher son 
venin au poison?

LE MAGICIEN. Non, Monseigneur. (FLAM, II.ii.78) par un lieutenant de police, a donné la mort à ce dernier après l'avoir fait blasphémer par trois fois. Suite à ce crime, qui n'est que justice - la justice corrompue protégeant le lieutenant -, Flamineo est allé voir un prêtre qui a refusé de l'absoudre. Ce refus le condamne à la damnation : " [...] je suis damné. Damné pour l'éternité » (FLAM, I.32). Dès lors, l'enfer n'est plus un possible auquel on peut échapper par une vie de vertu, l'enfer devient « une certitude ». Et cette certitude est cela même qui libère Flamineo de tout sens moral. Plus de garde-fou : " personne ne peut plus m'en faire une menace. Car, quoi que je fasse maintenant, je ne saurais être davantage damné que je ne suis » (FLAM, I.32). Cette épée de Damoclès ayant disparu, Flamineo est libre et le clame dans une tirade aux accents dostoïevskiens :

[...] fini la peur de l'enfer ! Fini l'immonde terreur de l'enfer qui vous torture tous, et un chacun, mes doux seigneurs, de la naissance à la mort! Je peux tout faire désormais! (Criant.) Je suis libre, moi! Je peux tout faire! Je peux mentir, trahir, voler, blasphémer, tuer. Je peux tuer n'importe qui ! Je peux tuer l'enfant à la mamelle ! Je peux tuer mon frère ! (FLAM, I. 33)

L'exemple est explicite : au lexique hyperbolique de la terreur, s'oppose l'irruption d'un " pouvoir » qui se clame, anaphorique, comme une revanche : "Je peux ». Au "vous » des soumis, des enchaînés à vie, s'oppose un « je » désaliéné et l'ouverture sur l'horizon 
de tous les crimes possibles, dans une cascade d'infinitifs - "mentir, trahir, voler, blasphémer, tuer " qui sont les négations de toutes les antiennes chrétiennes - qui s'achève sur le nefas, la sortie hors des limites de l'humanité, avec l'évocation d'actes parmi les plus inouïs, inhumains par excellence, l'infanticide et le fratricide.

Flamineo fait de la peur de l'enfer l'entrave, la seule entrave qui contraint les individus à tendre vers le bien. Et l'œuvre, comme le dit Aragon, devient « la dénonciation d'une morale qui n'a d'autre base que la crainte de l'enfer, où les hypocrites trichent avec le ciel, laissant à d'autres la matérialité du crime qui sert leurs intérêts ${ }^{28} »$. Et, si abject que soit Flamineo, «sa conscience s'oppose à cette hypocrisie ${ }^{29}$ ", comme le note Aragon.

Flamineo, c'est le monstre, certes, mais il est le produit hideux et sans masque d'une société corrompue. Or, pour que puisse demeurer la bonne conscience, le monstre doit être désigné, mon(s)tré, nommé. Et tous, dans l'œuvre, de faire bloc contre le « ruffian, proxénète, Caïn » (FLAM, IV.iii.132), contre l'« abominable » (FLAM, II.ii.79). Car, faire de l'assassin, du bourreau, d'un Flamineo, un monstre, un démon, est rassurant finalement : il n'est pas moi ; et il est rassurant aussi de ne pas le croire humain, comme il est rassurant de ne pas croire humains les Hœss et les Eichmann. Or, et c'est ce que suggère déjà Merle dans son Flamineo, c'est ce qu'il explorera plus avant dans La mort est mon métier, les Eichmann ne furent, comme le dit plus tard Hannah Arendt, ni pervers ni sadiques, mais terriblement et effroyablement normaux.

43 Merle est donc passé du "démon blanc ", une périphrase, à un nom propre, Flamineo, avant de pouvoir, à la première personne, donner une voix au bourreau : il y a là une véritable mouvement concentrique qui tente d'approcher au plus près l'individu, le criminel. Et s'il reste un jouissif fumet élisabéthain dans Flamineo, ce ne sera pas le cas dans La mort est mon métier. Car Merle, en deux ans, va explorer deux pôles antagonistes : après la monstration, le voyeurisme de l'effroi qu'il trouve chez Webster, qu'il exploite dans son Flamineo, il crée La mort est mon métier, l'anti-Webster, sans affect, sans pathos, sans corps sanglants et tordus, mais dans un style neutre, décolorant l'univers, plat, prosaïque.

Pour Aragon, sous la plume de Merle, par la réécriture de Webster, Flamineo devient un personnage représentatif $d u x^{e}$ siècle :

[...] un personnage complexe, le tueur tel que notre siècle l'a vu à Chicago ou à Shanghaï; le cagoulard ou le nazi, au service d'Hitler ou de l'Intelligence Service, non pas un homme de la Renaissance, mais un personnage de l'époque des guerres et des révolutions, qu'on appelle le xxe siècle, il n'y a pas à s'y tromper ${ }^{30}$.

C'est du détour par ce personnage tiré de Webster, c'est de la compréhension de ce monstre-là qu'a pu émerger le Rudolf Hœss du roman de Merle "qui n'eût certainement jamais écrit La mort est mon métier, ce portrait du SS Rudolf, s'il n'eût quelques années plus tôt tiré de Webster ce Flamineo [...] et appris dans Flamineo ce qui allait lui éclairer Rudolf ${ }^{31} »$. 


\section{NOTES}

1. Robert Merle, La mort est mon métier, [1952], Gallimard, coll. « Folio », Paris, 1972, préface, p. II.

2. John Webster, Le Démon blanc [The White Devil], traduction, introduction et notes par Robert Merle, Aubier, Paris, 1950. Toutes les pages indiquées entre parenthèses renvoient à cette œuvre et cette édition, abrégée comme suit (DB, Acte. Scène. Page) ; lorsqu'il n'y a nulle mention d'acte ou scène, la référence renvoie à l'introduction de Robert Merle.

3. Une chronique si «frappante » et si «italienne ", nous dit Merle, qu'elle attira l'attention de Stendhal qui l'évoque dans ses Chroniques italiennes (DB, 68).

4. Une biographie intitulée Vittoria, Princesse Orsini, publiée chez Del Duca en 1958 ; une nouvelle intitulée « Isabella », publiée dans la Revue de Paris en juillet de cette même année ; et un roman, L'Idole, publié en 1987.

5. Robert Merle, Flamineo - Sisyphe et la Mort - Les Sonderling, [1950], Gallimard, coll. « Le Manteau d'Arlequin », Paris, 1986. Toutes les pages indiquées entre parenthèses renvoient à cette œuvre et cette édition, abrégée comme suit (FLAM, Acte. Tableau. Page).

6. Louis Aragon, «Webster, Stendhal et Robert Merle », [Dans :] La lumière de Stendhal, Denoël, Paris, 1954, p. 119-161, p. 72.

7. Cité par Robert Merle (DB, 6).

8. Philarète Chasles, Études sur W. Shakespeare, Marie Stuart et l'Arétin. Le drame, les mœurs et la religion au XVI siècle, Amyot, Paris, 1852, p. 106, puis 107-108.

9. Line Cottegnies, François Laroque et Jean-Marie Maguin (dir.), Théâtre élisabéthain, T. 2, Gallimard, Bibliothèque de la Pléiade, Paris, 2009, p. 1700.

10. Line Cottegnies, François Laroque et Jean-Marie Maguin (dir.), Théâtre élisabéthain, op. cit., id.

11. Louis Aragon, «Webster, Stendhal et Robert Merle », op. cit., p. 121.

12. Louis Aragon, «Webster, Stendhal et Robert Merle », op. cit., p. 127.

13. Spectres dont Merle regrette qu'au $\mathrm{XX}^{\mathrm{e}}$ siècle ils ne fassent plus peur et que même ils fassent rire $(\mathrm{DB}, 26)$.

14. Pour Merle, Flamineo est un « Hamlet noir, affranchi une fois pour toute de ce "lait de la tendresse humaine" que le Prince de Danemark, comme Macbeth, conserve en lui » (DB, 71).

15. Comme l'indique Merle, le titre de Webster, périphrase désignant Vittoria, n'est pas sans évoquer le sermon prêché par T. Adams en 1613 à Saint-Paul et qui s'intitulait « The white divell, or the hypocrite uncased» (DB, 58). Et même si Webster n'emploie pas tout à fait l'expression dans le même sens, il n'en reste pas moins qu'elle a cours à son époque et qu'elle met l'accent sur une des grandes terreurs du XvII ${ }^{\mathrm{e}}$ siècle, l'hypocrisie.

16. Simplification qui entraîne, par exemple, la suppression des personnages de la mère vertueuse Cornelia et du cardinal Monticelso, futur pape.

17. Ainsi, au rêve de Vittoria qui suggérait d'éliminer les époux-obstacles chez Webster, Merle substitue le rêve de Flamineo, raconté à Brachiano pour l'inciter à soutenir le meurtre.

18. Louis Aragon, «Webster, Stendhal et Robert Merle », op. cit., p. 139.

19. Robert Merle, Flamineo, Corbeil-Essonnes : imprimerie CRÉTÉ, 29-4-1954, archives privées, préface, p. 1-2.

20. Robert Merle, Week-end à Zuydcoote [1949], Gallimard, coll. « Folio », Paris, 1972, p. 149.

21. Robert Merle, Nouveau Sisyphe - Justice à Miramar - L'Assemblée des femmes, Gallimard, coll. « NRF », Paris, 1957, p. 99.

22. Mais déjà, chez Webster, il se voit contraint de se parjurer en plein procès.

23. L'expression est celle-là même que Merle utilise pour qualifier Rudolf Hœss dans sa préface à La mort est mon métier.

24. Robert Merle, La mort est mon métier, op. cit., p. 324. 
25. Le Flamineo de Webster est, à n'en pas douter, l'« embryon de Lorenzaccio ", comme le signale au passage Beigbeder (Louis Aragon, «Webster, Stendhal et Robert Merle», op. cit., p. 122).

26. Ibid., p. 140.

27. Car, comme le dit très justement Aragon, « le texte anglais avance par images, mais se passe d'explications psychologiques » (Louis Aragon, «Webster, Stendhal et Robert Merle », op. cit., p. 140).

28. Ibid., p. 143.

29. Ibid., p. 144.

30. Louis Aragon, « Webster, Stendhal et Robert Merle », op. cit., p. 139.

31. Ibid., p. 142.

\section{RÉSUMÉS}

Cet article aborde le théâtre de Robert Merle et sa réécriture du Démon blanc de Webster. Qu'estce donc qui pousse le romancier $\mathrm{du} \mathrm{xx}^{\mathrm{e}}$ siècle à réécrire cette tragédie du sang toute baroque ? Qu'est-ce donc que Merle va chercher - et trouver - dans cette pièce macabre et morbide ? En quoi son Flamineo, au «fumet » élisabéthain, devient-il une pièce représentative $\mathrm{du} \mathrm{xx}^{\mathrm{e}}$ siècle? L'article aborde ces questions en deux temps : en montrant d'une part que l'univers de Webster propose une peinture effroyable et inégalée du mal ; d'autre part que, sous la plume de Merle, par la réécriture de Webster, Flamineo, ce " Hamlet noir ", devient un personnage représentatif du $\mathrm{XX}^{\mathrm{e}}$, et c'est du détour par ce monstre-là, qu'a pu émerger Rudolf Hœss, le bourreau d'Auschwitz, «terriblement et effroyablement normal », que Merle met en scène, à la première personne dans La mort est mon métier.

This article discusses Robert Merle's drama and his rewriting of Webster's White Devil. What brings the twentieth-century novelist to rewrite this tragedy of blood? What does Merle seek and find in this macabre and morbid play? What makes his Flamineo, an "Elizabethan" play, representative of the twentieth century? The article addresses these questions in two parts: first by showing that Webster's world provides a frightful and unparalleled painting of evil; then, that Merle turns Webster's Flamineo into a "black Hamlet", a character representative of the twentieth century - a detour which will pave the way for another monster, the "terribly and appallingly normal" executioner of Auschwitz, Rudolf Hoess, in Merle's first-person narrative Death Is My Trade.

\section{INDEX}

Mots-clés : Aragon, Démon, Effroi, Mal, Robert Merle, John Webster

Keywords : Aragon, Devil, Fright, Evil, Robert Merle, John Webster 


\section{AUTEUR}

\section{ANNE WATTEL}

Anne Wattel est agrégée et enseigne dans le secondaire. Elle est l'auteur d'une thèse de doctorat portant sur l'œuvre romanesque de Robert Merle, sous la direction de Nelly Wolf, à I'Université de Lille 3 - Charles de Gaulle (Univ. Lille, EA 1061 - ALITHILA - Analyses Littéraires et Histoire de la Langue, F-59000 Lille, France). 